\title{
Fatores genéticos e ambientais associados a espinha bífida
}

\author{
Genetic and ambient factors and profile of the newborns with spina bifida
}

Cristiane de Jesus da Cunha1, Tiago Fontana², Gilberto de Lima Garcias ${ }^{3}$, Maria da Graça Martino-Roth ${ }^{4}$

\section{RESUM0}

Objetivos: analisar a frequiência e os fatores associados à ocorrência da espinha bífida. Métodos: os dados foram obtidos por meio de entrevista de 47 casos e 47 controles, nascidos nas cinco maternidades da cidade de Pelotas, durante o período de 1 de Janeiro de 1990 a 31 de Dezembro de 2003. É estudo com delineamento de caso-controle, de base populacional que abrangeu todos os nascimentos hospitalares. O controle foi o neonato normal que nasceu após cada caso com malformação. Todos os dados foram obtidos mediante questionário-modelo. A análise do planejamento de análise de dados incluiu o uso do teste $t$ de Student, $\chi^{2}$ e odds ratio. Resultados: ocorreram aproximadamente 77.000 nascimentos nesse período. Desses, 1.043 (1,3\%) apresentaram algum tipo de malformação congênita. Dentre essas, 47 de 162 anomalias do fechamento do tubo neural foram diagnosticadas como espinha bífida. Foram encontradas diferenças significativas quanto ao número de natimortos prévios, bem como proporção superior de casos de espinha bífida em recém-nascidos do sexo feminino. Neste estudo, muitos fatores como o uso de medicamentos; doenças agudas; afecções crônicas; número de gestações; idade, escolaridade e ocupação dos pais, entre outros, não mostraram associação com o nascimento de recém-nascido com espinha bífida. Conclusões: a espinha bífida deve ser considerada como importante fator de risco para a morbidade perinatal, e sua ocorrência está associada a um histórico gestacional de natimortos prévios.

PALAVRAS-CHAVE: Disrafismo espinhal/genética; Anormalidades; Fatores de risco

\section{ABSTRACT}

Purposes: to analyze the frequency, associated risk factors for the occurrence of spina bifida and differences between the newborns carrying this malformation and the newborns' morbidities. Methods: data were obtained through interview of 47 cases and 47 controls, born in the five maternities of the city of Pelotas, during the period from January 1, 1990 to December 31, 2003. This is a population-based case-control study, comprising all births occurred in hospitals. The control was the normal newborn that was born after each case with spina bifida. All data were obtained by using the model ECLAMC questionnaire. The planning of analysis of data included the use of the Student's $t$ test, $\chi^{2}$ and odds ratio. Results: in this period there were approximately 77,000 births. Of these, 1,043 (1.35\%) presented some type of congenital malformation. Among these, 47 of 162 anomalies of the neural tube (29\%) were spina bifida cases. Significant differences have been found in the number of previous stillborn babies, as well as a higher rate of spina bifida cases in females. In this study, many factors such as the use of medical drugs; acute and chronic illnesses; number of gestations; age, education and occupation of the parents, among others, did not influence the occurrence of spina bifida. Conclusions: spina bifida must be considered as an important factor of risk for perinatal morbidity, and its occurrence is associated with a history of previous stillborn babies.

KEYWORDS: Spinal dysraphism/genetics; Abnormalities; Risk factors

\section{Introdução}

As malformações congênitas (MC) pesam de modo considerável nas estatísticas de morbidade e mortalidade perinatais devido ao aumento das taxas de incidência ${ }^{1}$. No Rio Grande do Sul, as MC são a segunda causa de morte em crianças com menos de um ano de vida, respondendo por cerca de $21 \%$ da mortalidade ${ }^{2,3}$. Como um grupo, os defeitos do tubo neural são causa importante de mortalidade neonatal, morte no início da

1 Pós-graduanda em Saúde Pública pelo Instituto Superior de Ensino, Pesquisa e Extensão de Pelotas - RS

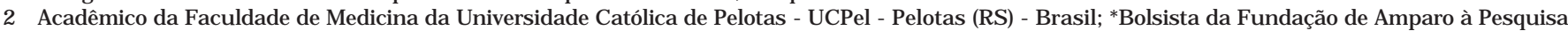
do Estado do Rio Grande do Sul

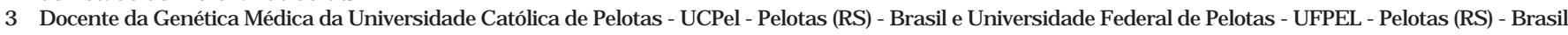

4 Docente da Genética Médica da Universidade Católica de Pelotas - UCPel - Pelotas (RS) - Brasil

Correspondência: Cristiane de Jesus da Cunha

Rua Dr. Cassiano no 620 - Centro - 96015-700 - Pelotas - RS - (53) 225-2048; 3028-2098; 9101-1319 - cunhacristiane@yahoo.com.br

Recebido em: 3/1/2005

Aceito com modificações em: 23/6/2005

Rev Bras Ginecol Obstet. 2005; 27(5): 268-74 
lactância e incapacidade nas crianças sobreviventes. Sua incidência populacional é variável desde $0,2 \%$ ou menos nos EUAa $1 \%$ na Irlanda ${ }^{3}$.

Nos casos de espinha bífida (EB) há falha na fusão dos arcos das vértebras, tipicamente na região lombar. Existem graus variáveis, desde a espinha bífida oculta, na qual o defeito é apenas no arco ósseo, à espinha bífida aberta, muitas vezes associada a meningocele (protrusão das meninges) ou meningomielocele (protrusão de elementos neurais além das meninges) ${ }^{3}$.

Esse defeito ocorre como conseqüência da associação de fatores genéticos e ambientais, sendo que muitas causas têm sido propostas, tais como deficiência de folato, diabetes materna, deficiência de zinco e ingestão de álcool durante os três primeiros meses de gravidez. Ainda a exposição materna a determinados medicamentos, como a carbamazepina e ácido valpróico, pode induzir à formação de tal defeito ${ }^{4}$. A prevenção da espinha bífida pode ser feita por meio da suplementação com $5 \mathrm{mg}$ de ácido fólico por dia durante o período periconcepcional (três meses antes da fecundação até 3 meses de gestação) ${ }^{5}$.

Em Pelotas, as malformações congênitas são a segunda maior causa de mortalidade em crianças de menos de um ano de idade, a terceira causa de morte em crianças de um a quatro anos e a quarta causa em crianças de cinco a quatorze anos de idade. As malformações congênitas foram responsáveis por $16,5 \%$ das mortes infantis no município de Pelotas, durante o ano de $2001^{6}$.

Este estudo teve como objetivo analisar a freqüência, os fatores predisponentes para a ocorrência da espinha bífida e mudanças do perfil dos neonatos portadores desta malformação.

\section{Métodos}

Este estudo foi baseado na análise de 47 casos (recém-nascidos portadores de EB) e de 47 controles (recém-nascidos desprovidos de morbidade) da cidade de Pelotas, RS, no período de 1 de janeiro de 1990 a 31 de dezembro de 2003.

O presente projeto teve seu início em 1990 e, desde então, vem sendo realizado, nos berçários de todos os hospitais de Pelotas. cidade do Rio Grande do Sul com aproximadamente 324.752 habitantes, segundo metodologia descrita no Manual Operacional ECLAMC-MONITOR (edição de 1982). São incluídos no programa todos os nascimentos, vivos ou mortos, de $500 \mathrm{~g}$ ou mais de peso, ocorridos nos hospitais a partir de seu ingresso no programa. Não se consideraram os nascimentos ocorridos fora do hospital e que posteriormente ingressaram no setor de neonatologia.
Todos os recém-nascidos, vivos ou mortos, foram examinados clinicamente por pediatras, segundo o protocolo de cada maternidade, em busca de malformações, e esta observação estendeu-se até sua alta hospitalar. Um segundo exame foi feito por acadêmicos do curso de Medicina, especialmente treinados para a detecção de anomalias congênitas. Os recém-nascidos portadores de qualquer tipo de malformação foram reexaminados por especialistas do projeto.

$\mathrm{Na}$ definição e descrição das malformações, considerou-se como malformação toda alteração morfológica, interna ou externa, clinicamente diagnosticável, com aceitável grau de certeza antes da alta hospitalar. Para cada recém-nascido com espinha bífida (RNEB), tomou-se um neonato vivo desprovido de morbidade (controle) pareado a ele. Foi controle o recém-nascido vivo não malformado e de igual sexo que nasceu no mesmo hospital imediatamente depois do malformado.

Para a formação do banco de dados foram realizadas a tabulação e codificação das informações obtidas, tais como peso, tipo de apresentação ao nascer, tipo de parto, tipo de alta do recém-nascido no momento da realização do questionário, informações sobre o pré-natal, exames ultrasonográficos, doenças agudas e crônicas, uso de medicamento durante a gestação, imunização materna, número de gestações, natimortos e abortos espontâneos prévios, idade, escolaridade e ocupação dos pais, consangüinidade, presença de malformações familiares e zona de moradia.

Os parâmetros utilizados para designar as variáveis relacionadas ao peso dos recém-nascidos foram: muito baixo peso, de 501 a 1.499 g; baixo peso, de 1.500 a $2.499 \mathrm{~g}$, e peso normal, acima de $2.500 \mathrm{~g}$. A gestante foi considerada portadora de anemia quando seu hematócrito foi inferior a 36\% nos exames laboratoriais solicitados. A hipertensão arterial sistêmica foi considerada como doença crônica, quando este fato já havia sido diagnosticado previamente à gravidez e a paciente referia valores para pressão arterial superior a 140/90 mmHg. Essa foi considerada como doença aguda, quando seu diagnóstico foi observado durante o período gestacional e considerados os mesmos valores acima descritos. Considerou-se consangüinidade toda e qualquer relação entre indivíduos de uma mesma linhagem hereditária. Foi designado que o baixo grau de escolaridade significou indivíduos analfabetos ou que tinham cursado até o ensino fundamental completo; médio grau para os que tinham até o ensino médio completo e alto grau para os que possuíam ensino superior e/ou técnico.

Quanto ao tipo de alta do recém-nascido, foram consideradas três variáveis: alta vivo, alta "morto" (óbito) e sem alta (termo encontrado no formulário e que foi utilizado no momento em que 
foi feita a entrevista, fator que podia ser alterado até um mês depois do nascimento do recém-nascido em questão, período que reflete a periodicidade com que as fichas foram e são coletadas nos hospitais por seus respectivos coordenadores).

As informações foram empregadas para o preenchimento dos formulários modelo ECLAMC e do programa SPSS for Windows versão 11.0. Para a análise dos dados foram utilizados o teste $t$ de Student, o $\chi^{2}$ e o odds ratio (OR; IC 95\%), sendo considerados significativos os resultados com $\mathrm{p}<0,05$. Os programas SPSS for Windows versão 11.0 e Epi-Info Versão 6.0 foram utilizados para o cálculo dessas análises.

As fontes bibliográficas utilizadas foram obtidas por meio de pesquisa em bancos de dados tais como Scielo, Bireme, Pub Med, New England Journal of Medicine, Blackwell Synergy.

O presente estudo foi aprovado pela Comissão de Ética em Pesquisa da Universidade Federal do Rio Grande do Sul.

\section{Resultados}

Durante os quatorze anos desta pesquisa, houve em Pelotas cerca de 77.000 nascimentos nos hospitais-maternidade. Nesse período, ocorreram 47 casos de RNEB o que determinou prevalência de aproximadamente 6 casos para cada 10.000 nascimentos.

Dentre os RNEB foi observado aproximadamente o dobro de casos do sexo feminino (proporção de 1,76:1 para sexo feminino e masculino, respectivamente). Constatou-se também que $8,5 \%$ dos RNEB nasceram mortos. Ao analisar o peso, verificou-se que este não diferiu entre casos e controles $(p=0,74)$, sendo verificado que, nos RNEB, $6,8 \%$ nasceram com muito baixo peso, $13,7 \%$ com baixo peso e $79,5 \%$ com peso normal (Tabela 1 ). Referente à sazonalidade, foi encontrado que 36,2 e $31,9 \%$ dos casos nasceram no verão e no inverno, respectivamente.

Não houve correlação significativa entre casos e controles, quanto à apresentação do neonato no momento do parto $(\mathrm{p}=0,08)$ (Tabela 1). Observou-se que 55,6 e 44,4\% dos RNEB e 66 e 31,9\% dos controles nasceram de parto normal e cesariana, respectivamente $(\mathrm{p}=0,31)$ (Tabela 1). Já quanto ao tipo de alta, no momento da realização do questionário, foi de 40,8 e 87,9\% para "alta vivo", 22,2 e $0 \%$ para "alta morto" e 37 e 12,1\% para "sem alta" nos casos e controles, respectivamente $\left(\mathrm{p}<0,001 ; \chi^{2}=16,23\right)($ Tabela 1$)$.
Tabela 1 - Características clínicas relacionadas ao nascimento dos recém-nascidos com espinha bífida e seus controles.

\begin{tabular}{|c|c|c|c|}
\hline Variável & $\begin{array}{l}\text { Casos }^{1} \\
n=47\end{array}$ & $\begin{array}{c}\text { Controles }^{1} \\
n=47\end{array}$ & $\chi^{2}(\mathrm{p}) ;$ ORB (IC 95\%) \\
\hline \multicolumn{4}{|l|}{$\mathrm{Peso}^{3}$} \\
\hline Muito baixo & $3(6,8 \%)$ & 0 & \\
\hline Baixo peso & $6(13,7 \%)$ & $7(15,6 \%)$ & $0,11(0,74)$ \\
\hline Normal & $35(79,5 \%)$ & $38(84,4 \%)$ & \\
\hline \multicolumn{4}{|c|}{ Tipo de apresentação } \\
\hline Cefálica & $26(81,3 \%)$ & $32(97 \%)$ & \\
\hline Podálica & $4(12,5 \%)$ & 0 & $4,94(0,08)$ \\
\hline Outra & $2(6,2 \%)$ & $1(3 \%)$ & \\
\hline \multicolumn{4}{|l|}{ Tipo de parto } \\
\hline Normal & $25(55,6 \%)$ & $31(66 \%)$ & \\
\hline Fórceps & 0 & $1(2,1 \%)$ & $2,31(0,31)$ \\
\hline Cesariana & $20(44,4 \%)$ & $15(31,9 \%)$ & \\
\hline \multicolumn{4}{|l|}{ Tipo de alta } \\
\hline Vivo & $11(40,8 \%)$ & $29(87,9 \%)$ & $16,23(0,0002)$ \\
\hline Morto & $6(22,2 \%)$ & 0 & \\
\hline Sem alta ${ }^{4}$ & $10(37 \%)$ & $4(12,1 \%)$ & \\
\hline
\end{tabular}

'Diferença em totais das variáveis devida à perda de informaç̃es.

"Valores de $\chi^{2}$ e odds ratio não presentes porque seus "limites" não foram considerados exatos quando calculados pelo programa Epi-Info.

3Muito baixo peso: 500 - 1.499 g; baixo peso: 1.500 - 2.499 g; peso normal: superior a $2.500 \mathrm{~g}$.

${ }^{4}$ No momento da entrevista o RN não havia, ainda, recebido alta.

Ao analisarem-se as variáveis relativas à mãe, constatou-se que $55,3 \%$ das mães dos casos (média de 7,6 consultas) e 53,2\% das mães dos controles (média de 7,4 consultas) freqüentaram pré-natal ( $\mathrm{p}=1$; OR=0,92). Dessas, 76,9 e 60,8\% das mães de RNEB e de controles foram submetidas à ultra-sonografia $(\mathrm{p}=0,36$; $\mathrm{OR}=0,47)$. Não houve diferença quanto à ocorrência de doença aguda durante a gestação entre os casos e seus controles $(\mathrm{p}=0,83 ; \mathrm{OR}=1,2)$. Entre as mães dos casos, as complicações mais freqüentes foram: infecção do trato urinário $(29,2 \%)$, anemia $(19,5 \%)$, infecção das vias aéreas superiores $(12,2 \%)$ e hipertensão gestacional $(7,3 \%)$. Foi observado que metade dessas doenças foram diagnosticadas no segundo trimestre da gestação, $32,2 \%$ no terceiro trimestre e $17,8 \%$ no primeiro trimestre.

Com a ocorrência de afecções crônicas, os resultados, da mesma forma, não diferiram significativamente entre mães de RNEB e de neonatos desprovidos de morbidade $(\mathrm{p}=0,66$; $\mathrm{OR}=1,48)$. Foi averiguado que, dentre as doenças crônicas encontradas nos casos, as mais prevalentes foram a hipertensão arterial sistêmica (50\%) e a bronquite (20\%). Verificou-se que $77,7 \%$ das mães dos casos e $65,2 \%$ das mães dos controles utilizaram algum tipo de fármaco durante o período gestacional $(p=0,27$; $\mathrm{OR}=1,87$ ), e que, nos casos, o uso desses ocorreu no terceiro trimestre da gravidez em 48,5; $33,3 \%$ no segundo trimestre e $18,2 \%$ no primeiro trimestre. 
Foi observado que 34,8 e $34,7 \%$ das mães de RNEB e controles foram vacinadas no decorrer da gravidez $(p=0,83$; $O R=1)$, sendo que, nas mães dos RNEB, essas vacinas foram realizadas em $64,3 \%$ no terceiro trimestre, em $21,4 \%$ no segundo e em $14,3 \%$ no primeiro.

Não foi detectada alteração expressiva no que se refere ao histórico de gestações entre casos e controles $(\mathrm{p}=0,42)$ (Tabela 2). Quanto ao número de natimortos prévios, verificou-se que $13,2 \%$ das mães dos RNEB haviam tido pelo menos um natimorto, ao passo que entre os controles, esse fato não foi observado $\left(\mathrm{p}=0,01 ; \chi^{2}=4,3\right)$. Quanto ao o número de abortos espontâneos prévios, $12,1 \%$ das mães dos casos e 4,7\% das mães dos controles relataram um ou mais abortos espontâneos $(\mathrm{p}=0,26)$.

Tabela 2 - Fatores genéticos, ambientais, antecedentes familiares e obstétricos dos recém-nascidos com espinha bífida e seus controles.

\begin{tabular}{|c|c|c|c|}
\hline Variável & $\begin{array}{l}\text { Casos }^{1} \\
(\mathrm{n}=47)\end{array}$ & $\begin{array}{l}\text { Controles }^{1} \\
(n=47)\end{array}$ & $\chi^{2}(\mathrm{p})$; ORB (IC 95\%) \\
\hline \multicolumn{4}{|l|}{ Número de gestações } \\
\hline Uma & $16(36,4 \%)$ & $15(32,6 \%)$ & \multirow[t]{4}{*}{$2,77(0,42)$} \\
\hline Duas & $13(29,5 \%)$ & $11(23,9 \%)$ & \\
\hline Três & $4(9,1 \%)$ & $10(21,8 \%)$ & \\
\hline Quatro ou mais & $11(25 \%)$ & $10(21,7 \%)$ & \\
\hline \multicolumn{4}{|l|}{ Idade materna } \\
\hline 13-19 anos & $10(22,7 \%)$ & $5(10,6 \%)$ & \multirow[t]{3}{*}{$(0,17)$} \\
\hline 20-34 anos & $29(65,9 \%)$ & $39(83 \%)$ & \\
\hline $35-60$ anos & $5(11,4 \%)$ & $3(6,4 \%)$ & \\
\hline \multicolumn{4}{|l|}{ Idade Paterna } \\
\hline 13-19 anos & $6(14 \%)$ & $5(10,6 \%)$ & \multirow[t]{3}{*}{$(0,15)$} \\
\hline 20-40 anos & $34(79 \%)$ & $42(89,4 \%)$ & \\
\hline $41-80$ anos & $3(7 \%)$ & 0 & \\
\hline \multicolumn{4}{|l|}{ Escolaridade materna ${ }^{3}$} \\
\hline Baixo grau & $24(60 \%)$ & $38(80,9 \%)$ & \multirow{2}{*}{$\begin{array}{c}3,63(0,05) ; \text { OR }=0,36 ; \\
\text { IC 95\%: } 0,12-1,03\end{array}$} \\
\hline Médio e alto graus & $16(40 \%)$ & $9(19,1 \%)$ & \\
\hline \multicolumn{4}{|l|}{ Escolaridade paterna $^{3}$} \\
\hline Baixo grau & $24(60 \%)$ & $37(78,7 \%)$ & \multirow{2}{*}{$\begin{array}{c}2,78(0,09) ; O R=0,4 \\
\text { IC 95\%: } 0,14-1,14\end{array}$} \\
\hline Médio e alto graus & $16(40 \%)$ & $10(21,3 \%)$ & \\
\hline \multicolumn{4}{|l|}{ Malformados na família } \\
\hline Sem & $36(81,8 \%)$ & $36(78,2 \%)$ & \multirow{2}{*}{$\begin{array}{c}0,03 \text { (0,87); OR=1,25; } \\
\text { IC 95\%: } 0,4-3,98\end{array}$} \\
\hline Com & $8(18,2 \%)$ & $10(21,8 \%)$ & \\
\hline Antepassados ${ }^{4}$ & $10(21,8 \%)$ & & \\
\hline Caucasóides & $41(82 \%)$ & $50(80,6 \%)$ & \multirow{2}{*}{$\begin{array}{l}0(0,95) ; O R=1,09 \\
\text { IC 95\%: } 0,38-3,16\end{array}$} \\
\hline Negróides & $9(18 \%)$ & $12(19,4 \%)$ & \\
\hline
\end{tabular}

${ }^{1}$ Diferença em totais das variáveis devida à perda de informações.

2Valores de $\chi^{2}$ e odds ratio não presentes porque seus "limites" não foram considerados exatos quando calculados pelo programa Epi-Info.

${ }^{3}$ Baixo grau: analfabeto - ensino fundamental completo; médio e alto graus: ensino médio incompleto - ensino superior.

${ }^{4} 0$ número nos casos e controles referentes a esse fator é maior devido às mães de ambos grupos relatarem mais de uma etnia para a variável antepassados.
Constatou-se maior número de RNEB em mães cuja faixa etária foi de 20 a 34 anos $(65,9 \%$ nos casos e em $83 \%$ nos controles $(\mathrm{p}=0,17)$. No que se refere à idade paterna, 14 e 10,6\% tinham entre 13 e 19 anos, 79 e $89,4 \%$ de 20 a 40 anos e 7 e $0 \%$ de 41 a 80 anos nos casos e controles, respectivamente $(\mathrm{p}=0,15)$ (Tabela 2$)$.

Foi verificado que $60 \%$ das mães dos RNEB e $80,9 \%$ dos controles tinham baixa escolaridade $\left(\mathrm{p}=0,05 ; \chi^{2}=3,63 ; \mathrm{OR}=0,36\right)$ (Tabela 2). Também observou-se que $75 \%$ das mães dos casos e $75,6 \%$ das mães dos recém-nascidos desprovidos de morbidades não exerciam atividade remunerada (do lar ou estudantes) ( $\mathrm{p}=0,77 ; \mathrm{OR}=1,33$ ).

Foi averiguado que $60 \%$ dos pais dos RNEB e $78,7 \%$ dos pais dos controles relataram baixa escolaridade $\left(\mathrm{p}=0,09 ; \chi^{2}=2,78 ; \mathrm{OR}=0,4\right)$ (Tabela 2). Verificou-se que $10 \%$ dos pais dos casos e $13,6 \%$ dos pais dos controles não exerciam atividade remunerada (estudantes) $(\mathrm{p}=0,77)$. Não houve correlação significativa entre consangüinidade e ocorrência de EB. Constatou-se que 18,2\% dos pais dos casos e $21,8 \%$ dos controles relataram presença de malformados na família ( $p=0,87 ; \mathrm{OR}=1,25)$.

Os antepassados dos progenitores de casos e controles foram divididos em duas categorias: caucasóides e negróides. Foi encontrado que 18 e $82 \%$ dos antepassados dos casos eram negróides e caucasóides, respectivamente. Não foram observadas diferenças significativas entre casos e controles para essa variável $(\mathrm{p}=0,95$; $\mathrm{OR}=1,09)$ (Tabela 2). Quanto ao local de moradia dos pais dos RNEB, 95,6\% residiam na zona urbana e apenas $4,4 \%$ na zona rural. Dos pais dos controles, 95,7\% moravam na urbana e $4,3 \%$ na rural $(p=0,1)$.

\section{Discussão}

Referente à freqüência da EB na população, valores tanto menores como maiores foram en$\operatorname{contrados}^{7,8}$. Tais diferenças podem ser devidas a fatores geográficos, socioeconômicos, sazonais e raciais, o que sugere complexa interação de múltiplos fatores ambientais e de fatores genéticos ${ }^{9,10}$.

Outras pesquisas também relataram maior freqüência de EB em neonatos do sexo feminino ${ }^{3,9,11}$. A explicação para esse fenômeno tem sido associada ao fato de que, para o fechamento do tubo neural, o feto feminino necessita maior quantidade de gonadotrofina coriônica humana que o feto masculino e alguma deficiência da função deste hormônio pode aumentar o risco para esse tipo de malformação ${ }^{12}$. As gonadotrofinas coriônicas podem ser detectadas no plasma e uri- 
na materna dez dias depois da ovulação, mas somente alcançam sua máxima concentração em 40 a 50 dias após a fecundação. Isso reforça essa hipótese, uma vez que o fechamento do tubo neural ocorre nas quatro primeiras semanas do desenvolvimento embriofetal ${ }^{7,13,14}$.

Defeitos do tubo neural (DTN), como o aqui analisado, são importante causa de natimortalidade fetal ${ }^{7}$. Apesar de este estudo não ter mostrado correlação entre o peso do recém-nascido e EB, outros demonstraram que baixo peso ao nascer é fator de risco não somente para a ocorrência de malformações do sistema nervoso central, mas também para risco aumentado de mortalidade neonatal ${ }^{7,15}$.

A freqüência na ocorrência das malformações congênitas pode variar de acordo com a estação de nascimento ${ }^{3}$. Assim como neste estudo, trabalhos em que a sazonalidade foi averiguada demonstraram maior freqüência de casos no verão ${ }^{16,17}$. O fato de um neonato ter EB não influenciou nos tipos de parto e na sua apresentação. Porém, essa malformação exerceu influência de forma bastante significativa no que se refere ao tipo de alta desses neonatos malformados. Portanto, a EB também parece atuar como importante fator de risco para a mortalidade perinatal.

Não foi detectada associação importante entre as variáveis pré-natal e ultra-sonografia com a EB. Porém, sabe-se que controles pré-natais deficientes (carentes de cuidados e informações) são considerados como fator de risco para a ocorrência de $\mathrm{DTN}^{18}$. Apesar de não ter sido encontrada correlação entre afecções agudas e EB, sabe-se que estas doenças exercem um papel importante no aparecimento deste defeito congênito ${ }^{14,18,19}$. Um fator que pode ter contribuído para isso foi o período de exposição fetal a essas doenças, fato considerado importante para o desenvolvimento embriofetal normal ${ }^{3}$. Não há evidência de que as doenças crônicas possam ser consideradas como um fator de risco para o surgimento de EB. Contudo, vários estudos mostraram que as afecções crônicas desempenham um papel importante no que se refere aos DTN em geral ${ }^{14,18,19}$.

Quanto ao uso de medicamentos, não foram encontradas diferenças significativas entre os dois grupos. Os fatores "período de exposição" e "tipos de medicamentos" podem explicar os resultados encontrados (o uso de medicamentos não atuou como fator de risco para a ocorrência de EB). Contudo, o uso de certos remédios, associado a um genótipo materno e/ou fetal propícios, e ainda somado à época de exposição, ao potencial teratogênico e à dosagem destes, pode agir de forma significativa para o aparecimento de DTN, como o aqui estudado ${ }^{3,18,19}$.
O fato de a mãe ter recebido vacinação durante o período gestacional não atuou como fator de risco nem como de proteção nesta pesquisa. Sua distribuição temporal em relação aos trimestres pode ter influenciado nesse resultado. Há, ainda, uma carência muito grande de estudos direcionados para essa área.

O número de gestações prévias não se mostrou relacionado ao nascimento de um RNEB. Porém, o fato de que $61,4 \%$ dos casos foram observados em mães primíparas e multíparas merece atenção. Rubin ${ }^{20}$ destacou que o risco para uma malformação congênita aumenta consideravelmente na primeira gestação e em mães com mais de seis gestações anteriores. Outros estudos averiguaram que a multiparidade é fator de risco para a ocorrência de um DTN ${ }^{7,11}$.

Houve correlação significativa entre número de natimortos prévios e nascimento de um RNEB. Provável explicação para isso reside em um componente genético, pois a grande maioria dos natimortos e abortos espontâneos são decorrentes de malformações embriofetais e, portanto, pode aumentar a probabilidade de recorrência dessa malformação em questão. Já foi observado por Stevenson et al. ${ }^{19}$ que mães com uma história positiva para essa variável têm risco maior de ter um filho malformado. Quanto ao número de abortos espontâneos prévios, não foi observada a correlação entre este fator e o aparecimento de EB. Não obstante, foi citado que a incidência dos DTN têm sido associada a uma maior freqüência de abortos espontâneos nas gestações anteriores ${ }^{19}$.

A idade materna foi outro dado que não diferiu entre os grupos e, portanto, não teve influência sobre a ocorrência de EB. Contudo, muitos estudos citam que mães de 13 a 19 anos e mães com 35 anos ou mais têm probabilidade maior de ter um filho com $\mathrm{EB}^{7,9,14,18,21}$. Do mesmo modo, não houve correlação entre idade paterna e EB. Rajab et al. $^{21}$ relataram que apenas a idade materna, e não a paterna, está relacionada com o surgimento de um RNEB. Em contraposição a esses achados, outros pesquisadores comprovaram que pais jovens e pais com idade avançada estão mais propensos a ter um filho com essa malformação ${ }^{18}$.

A escolaridade materna e paterna e a ocupação (fonte de renda) destes não diferiram entre casos e controles. Mas foi observado que a maioria das mães e dos pais dos RNEB tinham um baixo grau de instrução, a maioria das mães não tinha renda e grande parte dos pais tinha atividade remunerada (operários não qualificados e independentes formaram a maior parte destes casos). A soma de todos esses resultados leva a uma condição socioeconômica muito baixa e que, de acordo 
com outros estudos, traz uma situação de elevado risco para a ocorrência de DTN. Ainda, isso pode ser devido ao fato de que mães nessa faixa socioeconômica ingerem quantidades significativamente menores de proteínas, polissacarídeos, fibras, ferro e niacina, o que aumenta o risco para a ocorrência de $\mathrm{EB}^{4,9,22,23}$.

A consangüinidade foi outra variável que não exerceu influência no nascimento de RNEB, e este fato é corroborado pelos achados de Rubin ${ }^{20}$. Porém, algumas pesquisas relataram que a consangüinidade é fator de risco para o aparecimento de DTN ${ }^{18,21,24}$. Com base nos dados obtidos com relação à presença de malformados na família, inferiu-se que este fator não influiu na ocorrência de RNEB. Contudo, estudos mostram que pode existir influência genética e que sua transmissão pode ser do tipo autossômica recessiva. Além disso, o risco de recorrência estimado para os DTN é de aproximadamente 5\% e tende a ser maior para o sexo feminino ${ }^{7,14}$.

Não foi verificada associação entre antepassados e aparecimento de EB, fato este também observado por outros, os quais relataram que a raça não foi significativamente correlacionada com os defeitos do tubo neural ${ }^{9}$. Porém, o risco de ocorrência de algumas malformações parece variar de acordo com a miscigenação ocorrida entre os grupos étnicos ${ }^{3,25}$. No que se refere à zona de moradia, não foi obtida associação entre esta e a ocorrência de $\mathrm{EB}$, fato já relatado anteriormente. No entanto, estes mesmos autores relataram uma maior proporção de casos na zona rural e não na zona urbana, como neste estudo.

Portanto, concluiu-se que neonatos do sexo feminino estão mais propensos a desenvolver espinha bífida do que recém-nascidos do sexo masculino. Ademais, esse defeito congênito deve ser considerado como importante fator de risco para a morbidade perinatal e sua ocorrência é comprovadamente associada a um histórico gestacional de natimortos prévios e relacionada com baixa condição socioeconômica, o que pode sugerir nutrição deficiente. Os cuidados na dieta materna, bem como o uso de ácido fólico complementar a esta, além de pesquisa rigorosa durante a anamnese, tornam-se importantes fatores para eventual proteção.

\section{Referências}

1. Penchaszadeh VB. Genetic services in Latin America. Community Genet. 2004;7(2-3): 65-9.

2. DATASUS. CID 10 - Malformações congênitas, deformidades e anomalias cromossômicas: espinha bífida [texto na Internet]. Brasília (DF): Ministério da Saúde [citado 2005 Maio 23]. Disponível em: http://www.datasus.gov.br.

3. Nussbaum RL, McInnes RR, Willard HF. Genética médica Thompson \& Thompson. 6a ed. Rio de Janeiro: Guanabara Koogan; 2002.

4. McDonnell R, Johnson Z, Doyle A, Sayers G. Determinants of folic acid knowledge and use among antenatal women. J Public Health Med. 1999;21(2):145-9.

5. Wald NJ, Law MR, Morris JK, Wald DS. Quantifying the effect of folic acid. Lancet. 2001;358(9298):206973.

6. Prefeitura Municipal de Pelotas. Plano municipal de saúde 2003-2004. Pelotas: Secretaria Municipal de Saúde e Bem Estar; 2003.

7. Nazer J, López-Camelo J, Castilla EE. ECLAMC: estudio de 30 años de vigilancia epidemiológica de defectos de tubo neural en Chile y en Latinoamérica. Rev Med Chil. 2001;129(5):531-9.

8. Sipek A, Gregor V, Horacek J, Masatova D. [Survival of children born with selected types of birth defects in Czech Republic]. Ceska Gynekol. 2004;69 Suppl 1:47-52.

9. Farley TF, Hambidge SJ, Daley MF. Association of low maternal education with neural tube defects in Colorado, 1989-1998. Public Health. 2002;1 16(2):89-94.

10. Botto LD, Moore CA, Khoury MJ, Erickson JD. Neural tube defects. N Engl J Med. 1999;341(20):1509-19.

11. Rittler M, López-Camelo J, Castilla EE. Sex ratio and associated risk factors for 50 congenital anomaly types: clues for causal heterogeneity. Birth Defects Res A Clin Mol Teratol. 2004;70(1):13-9.

12.Janerich DT. Female excess in anencephaly and spina bifida: possible gestations influences. Am J Epidemiol. 1975; 101(1):70-6.

13. Moore KL, Persaud TVN, Shiota K. Embriologia clínica. 6a ed. Rio de Janeiro: Guanabara Koogan; 2000.

14. Nazer J, Cifuentes L, Rodriguez M, Rojas M. Malformaciones del sistema nervioso central en el Hospital Clínico de la Universidad de Chile y maternidades chilenas participantes en el Estudio Colaborativo Latinoamericano de Malformaciones Congénitas (ECLAMC). Rev Med Chil. 2001;129(10):1163-70. 
15.Tuli S, Tuli J, Drake J, Spears J. Predictors of death in pediatric patients requiring cerebrospinal fluid shunts. J Neurosurg. 2004;100(5 Suppl Pediatrics):442-6.

16. Pei LJ, Li Z, Li S, Hong SX, Ye RW, Chen X, et al. The epidemiology of neural tube defects in high-prevalence and low-prevalence areas of China. Zhonghua Liu Xing Bing Xue Za Zhi. 2003;24(6):465-70.

17. Nugud A, Arbab M, Osman TM. Pattern of neural fusion defects in Sudan. Saudi Med J. 2003;24:S54.

18. Cortés F. Prevención primaria de los defectos de cierre del tubo neural. Rev Chil Pediatr. 2003;74(4):208-12.

19. Stevenson RE, Hall JG, Goodman RM. Human malformations and related anomalies. New York: Oxford University Press; 1993. v. 2.

20. Rubin A. Handbook of congenital malformations. Philadelphia: W. B. Saunders; 1967.
21. Rajab A, Vaishnav A, Freeman NV, Patton MA. Neural tube defects and congenital hydrocephalus in the sultanate of Oman. J Trop Pediatr. 1998;44(5):300-3.

22. Groenen PM, van Rooij IA, Peer PG, Ocke MC, Zielhuis GA, Steegers-Theunissen RP. Low maternal dietary intakes of iron, magnesium, and niacin are associated with spina bifida in the offspring. J Nutr. 2004;134(6):1516-22, 2004.

23. Wasserman CR, Shaw GM, Selvin S, Gould JB, Syme SL. Socioeconomic status, neighborhood social conditions, and neural tube defects. Am J Public Health. 1998;88(11):1674-80.

24. Murshid WR. Spina bifida in Saudi Arabia: is consanguinity among the parents a risk factor? Pediatr Neurosurg. 2000;32(1):10-2.

25. Yang J, Carmichael SL, Kaidarova Z, Shaw GM. Risks of selected congenital malformations among offspring of mixed race-ethnicity. Birth Defects Res A Clin Mol Teratol. 2004;70(10):820-4. 